\title{
An Encounter-based Routing Scheme for Delay Tolerant Networks
}

$$
\text { Hang GUO }{ }^{a} \text {, Bo SUb }
$$

Department of Aeronautical Armament Engineering, The First Aviation Academy of Chinese Air Force, Xinyang, 464000, China

aguohang0001@126.com, bsoboyhy@126.com

Keywords: DTNs;Routing algorithms; Contact capacity; Encounter angle

\begin{abstract}
Delay Tolerant Networks (DTNs) are a type of networks which suffer from intermittent disruption due to the factors such as mobility and energy. Whereas only a little state of art routing algorithms in DTNs address the problem of message loss due to the insufficient contact capacity because of mobility. In this paper, to reduce these aborted messages due to fast mobility we propose a scheme that adopts two separate routing algorithms. Specifically, only one of them is activated according to the current encounter angle. Besides, before the final delivery, the copies of the message which has been carried by most of the nodes in the network are likely to be cleared out after custody transfer. Simulation results exhibit our proposed algorithm achieve a higher delivery ratio with lower overhead.
\end{abstract}

\section{Introduction}

Delay Tolerant Networks (DTNs) [1] is designed to cope with the challenge condition in the restricted networks with sparse density, intermittent disruption and limited energy. By means of the store-carry-forward mechanism, the messages are relayed by the per hop behavior. Importantly, routing is the main challenge in DTNs since the characteristic of DTNs determines that the routing techniques in traditional networks cannot work effectively, which is extremely difficult to determine the potential end to end path towards the destination.

Contact capacity as an important factor affects the routing performance, which is rarely taken into account. Due to the high mobility the successful custody transfer cannot be finished under some condition thus the transmission cost for the corresponding message is wasted. Upon this problem the overhead and battery consumption would increase and these metrics are critical in the restricted scenario. One feasible approach to overcome this problem is to transmit less number of messages to the neighbor node when the current communication angle is large because the potential contact duration is limited. Relatively, to transmit more messages when this angle is small due to the longer contact duration is acceptable.

In the following section, we briefly review the current state of art routing algorithms in DTNs, and then we propose our algorithm. Based on the simulation result under realistic scenario, we draw the conclusion and present our future work.

\section{Related works}

The early stage of routing algorithms in DTNs are based on the single copy based forwarding mechanism [2][3], which forward the message to the candidate nodes with better utility value but only one copy of message exists in the network. Inherently, the performance of this approach is affected by the limited message life time. In general, the multi copy based replication mechanism can cope with this limitation but the overhead is significantly high due to the large number of redundancy [4]. Motivated by this drawback, the utility based replication scheme [5] is proposed to alleviate such overhead.

To our knowledge, the algorithms predict the potential contact duration according to angle is only proposed in [6] [7]. However, their analyses are only limited by two factors. Firstly, the prediction is based on the encounter angle and assumes this pair of encountered nodes would constantly keep this encounter angle until the contact is disrupted, which might not be realistic under some condition. 
Secondly, the analysis of encounter angle is only limited under the case when the encounter angle is less than $\pi / 2$. There assumption is not appropriate if this angle is larger than or equal to $\pi / 2$. Based on the above two aspects, to predict the potential contact duration by the angle when pair of nodes encounter is not feasible. In particular, we propose an approach based on the communication angle when the corresponding message is going to be transmitted, which is more simple but effective.

Buffer management plays an important role in DTN routing since the messages suffer from the long delay have to be kept in the limited buffer space until the contact is available. Therefore, both the priorities used for message to be transmitted and dropped when the buffer space is exhausted significantly affect the performance. Several existed routing algorithms have adopted such mechanism so far. Furthermore, once the message is delivered by the destination, the final receiver generates an acknowledgement and floods this information of which the size is quite small compared with the size of message [8] [9] to reduce the redundancy.

\section{The encounter-based routing scheme}

Our proposed algorithm is designed based on the following assumptions:

1: each node can only obtain its location by the GPS system, which is more realistic in reality. When pair of nodes are in communication, by the exchanged signaling information they can calculate the communication angle between each other.

2: the transmission range is quite small compared with the area of the scenario, therefore it is imprecisely to get the most recent location of destination by the broadcast information.

2: the mobility pattern is unpredictable and movement never stops even if pair of nodes are in communication.

3: due to the high mobility factor, the contact capacity is not enough for all of the requested messages.

The overall functions flow chart of our proposed algorithm is illustrated in Fig.1 and they are specifically introduced in the following parts of this section.

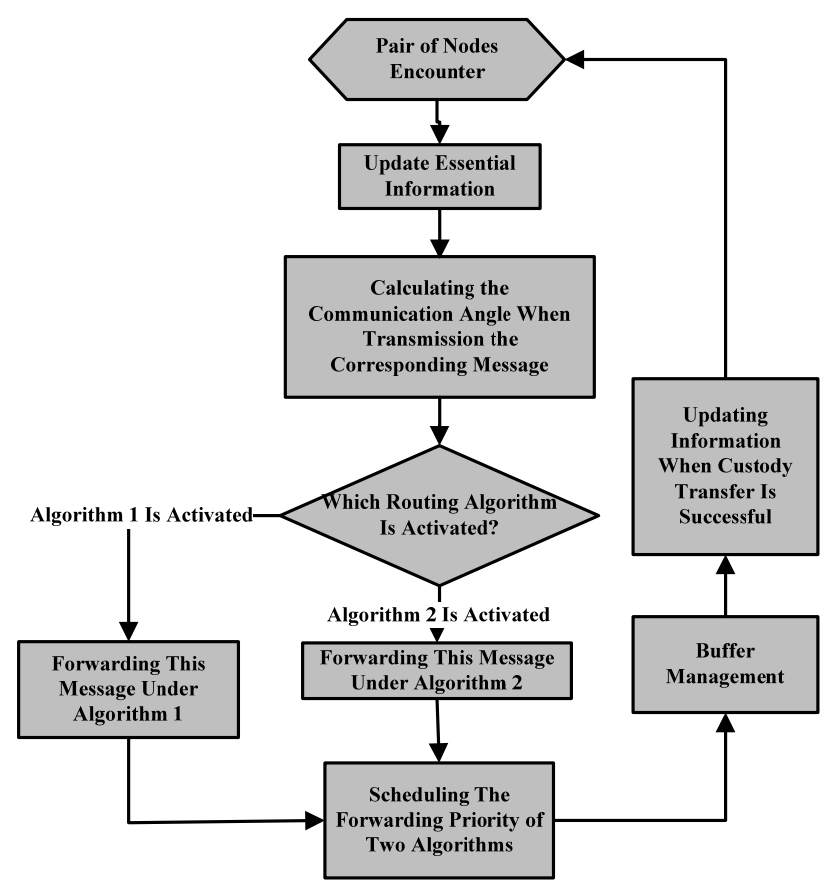

Fig.1. Functions flow chart of proposed algorithm

\section{Calculation of the Communication Angle}

Based on our assumptions, each node can obtain its current location by means of GPS system. With an appropriate time window $\mathrm{T}$ for the location updating, this approach is useful to predict the trajectory of the node. We define the mobility vector to indicate the current trajectory. 
Specifically, $\Delta V_{\mathrm{i}}$ is the variation of the mobility vector of arbitrary node $\mathrm{i}$ in the last $\mathrm{T}$, which consists of the specific variation of $\Delta X_{i}$ and $\Delta Y_{i}$. When pair of nodes are within each other's communication range, the communication angle $\Phi$ of them is calculated by Algorithm 1.

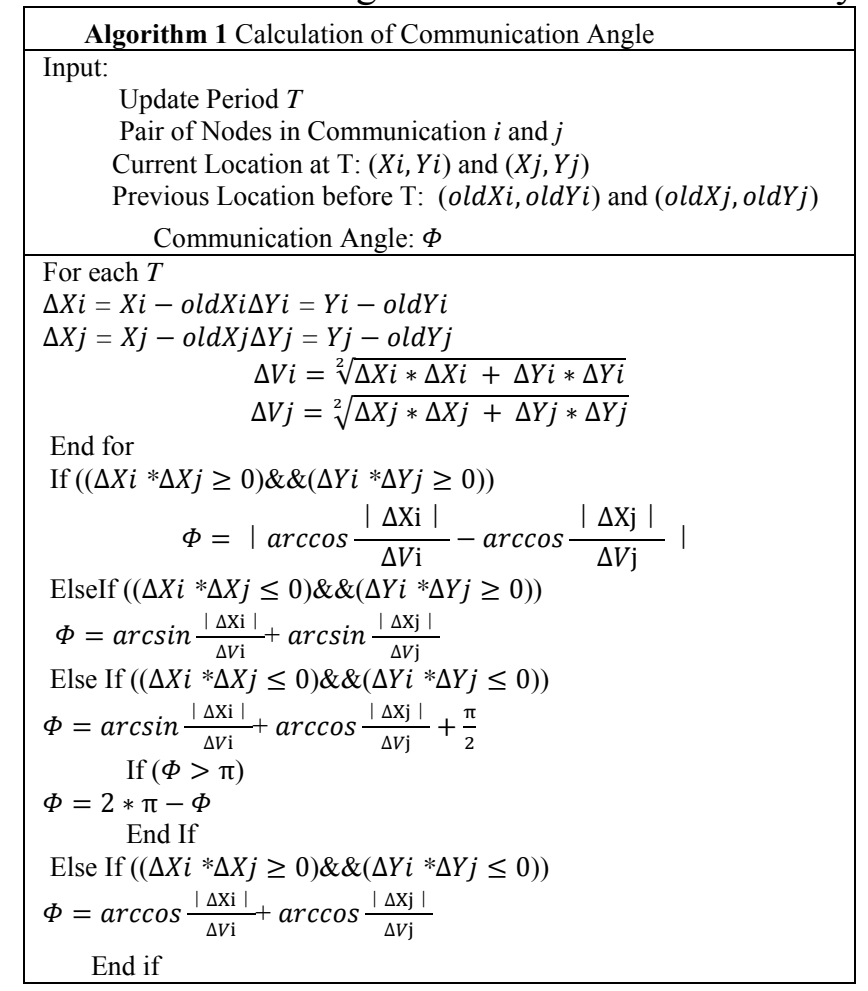

\section{Asynchronous Forwarding Framework}

Based on the previous calculation of communication angle, we focus on the asynchronous forwarding framework in this part. Generally, if this angle is quite small, accordingly the potential contact duration is considered to be longer than the case when this angle is very close to $\pi$. Fig.2 shows two particular examples. When arbitrary nodes $i$ and $j$ are in communication with the angle of 0 or $\pi$. The potential contact duration under the case of angle 0 is $2 R /|\operatorname{speed}(A)-\operatorname{speed}(B)|$ and the potential contact duration under the case of angle $\pi$ is $2 R /|\operatorname{speed}(A)+\operatorname{speed}(B)|$, where $R$ is the minimum transmission range of $\left[R_{a}, R_{b}\right]$ between these two nodes with respective movement speeds Speed $(A)$ and $\operatorname{Speed}(B)$.
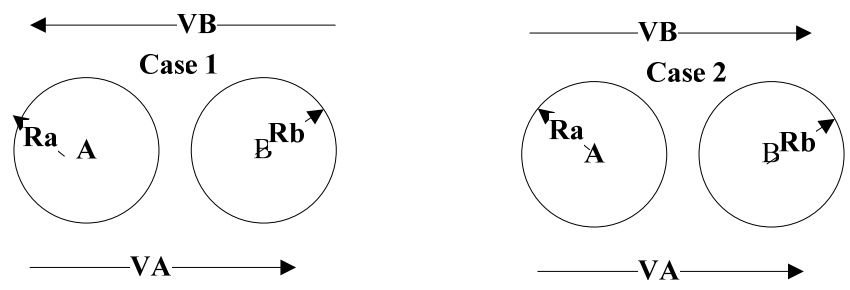

Fig.2. Prediction the potential contact duration under two communication angles

Compared with the analysis in related work, which can not precisely predict the potential contact duration. Our proposed approach is effective since the calculation of communication angle dynamically represents the current state. Accordingly, we propose to transmit less number of messages when the current communication angle is large and transmit more messages when the angle is small. The key question comes here is to transmit the most appropriate message to fully utilizes the bandwidth. Another benefit from this scheme is to reduce the number of aborted message due to mobility factor, which can reduce the energy consumption and generate less number of messages into the networks with the purpose of congestion avoidance.

However, ideally the naive flooding scheme can achieve better delivery ratio than the utility based replication scheme though it encounters the high overhead ratio, thus to implement the naive flooding mechanism when the communication angle is small can compensate the delivery ratio. In particular, 
though the message forwarding is not based on the delivery potential of encountered node, we still need a mechanism to optimize this mechanism.

For simplicity, we propose to transmit the message which has not been extensively flooded into the network with higher probability, hop count is one of the approaches to estimate this state of message. However it can not precisely estimate the practical situation of the messages in the network. Firstly, each node would not receive any incoming message that is being carried. Secondly, it might receive the same message again provided it dropped this message in the past and hop count of the corresponding message is increased by one under this case. Based on these limitations, we propose to use the carrier count which is increased by one when the corresponding message is received by the nodes with different ID.

Regarding the improved naive flooding scheme, we define the replication probability (RP) by Equation (1) and the specific algorithm of our improved naive flooding is illustrated in Algorithm 2.

$$
R P=1-\frac{\text { number of carriers in carrier count }}{\text { number of total nodes }}
$$

As we presented previously, the communication angle determines which forwarding algorithm is activated. We define an activated probability (AP) for this purpose by Equation (2) and the specific asynchronous framework is illustrated by Algorithm 3 .

$$
A P=\frac{\text { communication angle }}{\pi}
$$

\begin{tabular}{|c|c|}
\hline Algorithm 2 Improved Naive Flooding & Algorithm 3 Asynchronous Forwarding Framework \\
\hline $\begin{array}{l}\text { Input: } \\
\text { Current Carrier: i } \\
\text { Encountered Carrier: } \mathrm{j} \\
\text { Occupied Messages in Carrier i: M0,M1,......Mn }\end{array}$ & $\begin{array}{l}\text { Input: } \\
\text { Current Carrier: } i \text { with } I D_{i} \\
\text { Encountered Node: } j \\
\text { Occupied Messages in Carrier } i: M_{0}, M_{1}, \ldots \ldots M_{n} \\
\text { Utility Information in } M_{n} U_{n} \\
\text { Carrier Count in } M_{n}: C_{n} \\
\text { The Utility Value for the Destination of } M_{n} \text { on behalf of } i: T_{n}\end{array}$ \\
\hline $\begin{array}{l}\text { For Each Contact Between } \mathrm{i} \text { and } \mathrm{j} \\
\text { For Each Mn } \\
\text { If ( } \mathrm{j} \text { Already Has Mn) } \\
\text { Continue } \\
\text { Else } \\
\text { Generating a Random Value Ran } €(0,1) \\
\text { If (RP>Ran) } \\
\text { Replicate a Copy of Mn } \\
\text { End If } \\
\text { End If } \\
\text { End For } \\
\text { End For }\end{array}$ & $\begin{array}{l}\text { Initialization } \\
\text { For Each Generated } M_{n} \text { in } i \\
U_{n}=T_{n} \\
\text { Add } I D_{i} \text { into } C_{n} \\
\text { Initialization Another Parameters for } M_{n} \\
\text { End For } \\
\text { Contact Process } \\
\text { For Each Contact Between } i \text { and } j \\
\text { For Each Message } M_{n} \\
\text { Calculating the Communication angle } \\
\text { Generating a Random Value Ran } \epsilon(0,1) \\
\text { If (AP }>\text { Ran) } \\
\text { Forwarding } M_{n} \text { By Delegation Forwarding } \\
\text { Else } \\
\text { Forwarding } M_{n} \text { By Improved Naive Flooding } \\
\text { End If } \\
\text { End For } \\
\text { End For }\end{array}$ \\
\hline
\end{tabular}

\section{Simulation results}

The simulations are implemented by the ONE [10] simulator, we present the performance of Epidemic, Prophet and MaxProp and the proposed encounter based scheme.

Table 1 Simulation configuration

\begin{tabular}{l|l}
\hline Parameter & Value \\
\hline Simulation Time & $43200 \mathrm{~s}$ \\
Connectivity & Bandwidth: $2 \mathrm{Mb} / \mathrm{s}$ \\
& Transmission Range: $20 \mathrm{~m}$ \\
& Generating Interval: $30 \mathrm{~s}$ \\
Message & Size: $1 \mathrm{MB}$ \\
& Initial TTL: 360 Minutes \\
Buffer Size & $100 \mathrm{MB}$ \\
Number of Nodes & 200 \\
\hline
\end{tabular}


Regarding the delivery ratio in Fig.3, our proposed algorithm nearly achieves the highest performance which is close to MaxProp even if Maxprop replicates messages to arbitrary encountered node without utility limitation, which means our algorithm generates less number of copies than MaxProp but still achieves high delivery ratio. Compared with other algorithms, our algorithm with FIFO still keeps a better performance though is only a little lower than adaptive Epidemic.
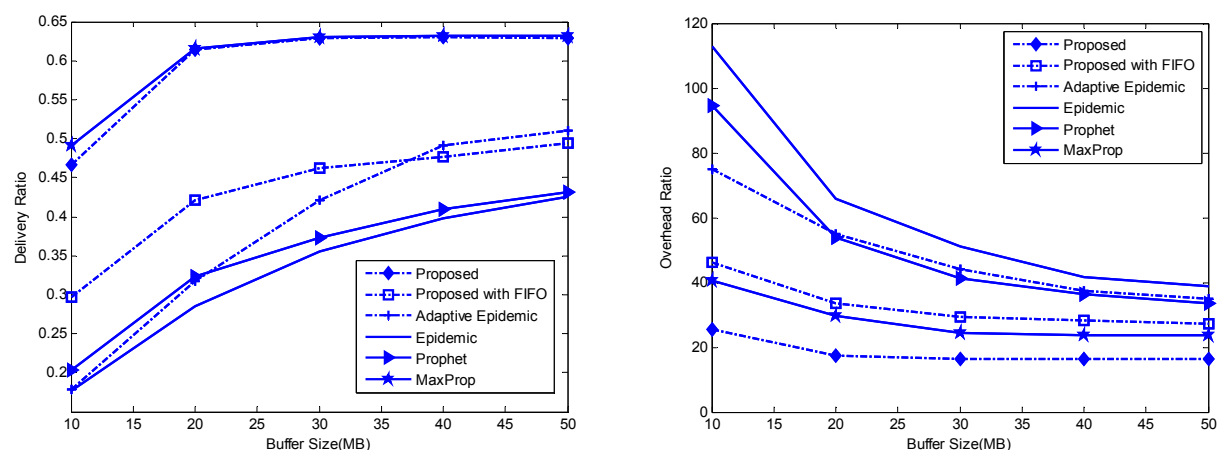

Fig.3. Buffer size vsdelivery ratio Fig.4. Buffer size vsoverhead ratio

In Fig.4, it is significantly that our proposed algorithm maintains the lowest overhead ratio. One of thecontributions is that dynamically determines the corresponding routing algorithm according to the current communication angle, which reduces the aborted messages potentially. The buffer management also improves the overhead ratio by determining the message forwarding and dropping. Without the buffer management, the overhead ratio of our algorithm still outperforms others except MaxProp that is designed with this function.

The average latency of our proposed algorithms is a tradeoff between MaxProp and other algorithms in Fig.5. Particularly, without the buffer management our algorithm maintains the latency whereas the latency of other algorithms which without buffer management as well is increased. The lower latency is beneficial though this metric is not important in DTNs since its application is regarded to be tolerant to the long latency.

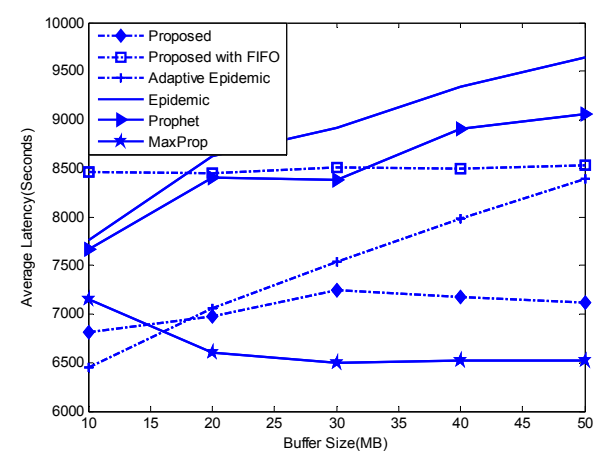

Fig.5. Buffer size vsaverage latency

\section{Conclusion}

Taking into account the potential contact capacity by the mobility issue is still in the early stage even if most of the routing algorithms in DTNs have been proposed in the last few years. Besides, the asynchronous forwarding framework can adaptively adjust the current routing algorithm to optimize the overall performance. In this paper, we address the potential contact duration issue and propose a simple framework based on the communication angle. Two routing algorithms are designed for the decision based on the communication angle. Our proposed algorithm not only reduces the number of aborted messages due to mobility factor but also achieves the significant performance compared with other state of art algorithms. In the future, we would extend our work with more intelligent mechanism for the asynchronous forwarding framework and further optimize the estimation of potential contact capacity. 


\section{References}

[1] Fall K, Farrell S. DTN: an architectural retrospective[J]. IEEE Journal on Selected Areas in Communications, 2008, 26(5):828-836.

[2] Jain S, Fall K R, Patra R K. Routing in a delay tolerant network[J]. ACM SIGCOMM Computer Communication Review, 2004, 34(4):145-158.

[3] Daly E M, Haahr M. Social network analysis for routing in disconnected delay-tolerant MANETs[C]// Proceedings of the 8th ACM international symposium on Mobile ad hoc networking and computing, 2007:32-40.

[4] Vahdat A, Becker D. Epidemic Routing for Partially-Connected Ad Hoc Networks[J]. Master Thesis, 2000.

[5] Lindgren A, Doria A, Schelén O. Probabilistic Routing in Intermittently Connected Networks[J]. Sigmobile Mobile Computing \& Communication Review, 2004, 7(3):19 - 20.

[6] G Sandulescu, S N Tehrani. Opportunistic DTN Routing with Window-aware Adaptive Replication.[C]// InProc of ACM AINTEC'08, Bangkok, November,2008.

[7] Zhuoqun Li, Lingfen Sun, Ifeachor, E.C. WSN10-5: Adaptive Multi-Copy Routing for Intermittently Connected Mobile Ad Hoc Networks[C]// Global Telecommunications Conference, 2006. GLOBECOM '06.IEEEIEEE, 2006:1 - 6.

[8] Balasubramanian A, Levine B N, Venkataramani A. Replication Routing in DTNs: A Resource Allocation Approach[J]. Networking IEEE/ACM Transactions on, 2010, 18(2):596 - 609.

[9] J Burgess, B Gallagher, D Jensen, B Levine. MaxProp: Routig for Vehicle-Based Disruption Tolerant Networking.[C]// In Proc of INFOCOM 2006. Barcelona: IEEE Communications Society, April 2006: 1-11.

[10] A Keränen, J Ott, T Kärkkäinen. The ONE Simulator for DTN Algorithm Evaluation[C]// SIMUTools'09: 2nd International Conference on Simulation Tools and Techniques. Rome, March 2009. 